\title{
Intermolecular structure factors of macromolecules in solution: Integral equation results
}

\author{
M. Fuchs ${ }^{1}$ and M. Müller ${ }^{2}$ \\ ${ }^{1}$ Physik-Department, Technische Universität München, 85747 Garching, Germany \\ ${ }^{2}$ Institut für Physik, Johannes Gutenberg-Universität Mainz, 55099 Mainz, Germany
}

(Received 12 February 1999)

\begin{abstract}
The intermolecular structure of semidilute polymer solutions is studied theoretically. The low-density limit of a generalized Ornstein-Zernicke integral equation approach to polymeric liquids is considered. Scaling laws for the dilute-to-semidilute crossover of the random-phase approximation (RPA)-like structure are derived for the intermolecular structure factor on large distances when intermolecular excluded volume is incorporated at the microscopic level. This leads to a nonlinear equation for the excluded volume interaction parameter. For macromolecular size-mass scaling exponents $\nu$ above a spatial-dimension dependent value, $\nu_{c}=2 / d$, meanfield-like density scaling is recovered, but for $\nu<\nu_{c}$ the density scaling becomes nontrivial in agreement with field-theoretic results and justifying phenomenological extensions of the RPA. The structure of the polymer mesh in semidilute solutions is discussed in detail and comparisons with large-scale Monte Carlo simulations are added. Finally, a possibility to determine the correction to scaling exponent $\omega_{12}$ is suggested.

[S1063-651X(99)01408-7]
\end{abstract}

PACS number(s): 61.25.Hq, 61.12.Ex, 61.20.Ja

\section{INTRODUCTION}

Whereas the conformational statistics of a single flexible polymer chain in dilute and semidilute solutions are understood rather well, less is known about the intermolecular packing. It is well understood that a semidilute polymeric solution builds up a temporary mesh with a mesh size, the density screening length $\xi_{\rho}$, which for macromolecular solutions can become large compared to the length scales characterizing the individual monomers $[1,2]$. However, the intermolecular packing, inside the mesh but still on length scales large compared to the chemistry-dependent local length scales, is as yet unclear. It has been the focus of recent neutron scattering experiments $[3,4]$, scaling considerations and field-theoretic calculations [4,5], and of computer simulations [6]. Older theories for the intermolecular structure, which either used the random-phase approximation (RPA) [7] or the assumption of Gaussian intermolecular correlations [8], failed to incorporate the non-mean-field-like correlations on scale $\xi_{\rho}$ of semidilute polymer solutions. The recent fieldtheoretic results lead to contradicting results as will be pointed out and resolved in this paper.

Integral equation theories for simple liquids directly address the problem of interparticle packing in dense fluids. Starting with the work of Schweizer and Curro [9], this approach has successfully been extended to macromolecular liquids. The polymer reference interaction site model (PRISM) integral equations have been fruitfully applied to describe inter alia the intermolecule correlations in dense homopolymer systems, polymer blends, and block copolymer melts [10]. PRISM is a macromolecular generalization of the reference interaction site model theory of small molecules of Chandler and Andersen [11,12]. The low-density limit of PRISM theory shall be worked out in detail in this paper in order to discuss the density correlations on the mesh size length scale. This paper either extends [13-15], or complements $[16,17]$ previous studies.

It is a priori not related to nor required for the success of the PRISM approach to polymer melts whether it also correctly captures the long-ranged correlations of semidilute polymer solutions. As liquid correlations in melts generally are short-ranged, an approach like PRISM appropriate for dense systems need not be a useful approach to (semi) dilute solutions, where long-ranged correlations are of interest. Nevertheless, the simplification of the PRISM equations to low polymer densities worked out here will be argued to provide a useful description of the intermolecular correlations building up the polymer mesh in polymer solutions $[10,13,14]$. Criteria for the quality of the approach will be established from comparisons with simulations, field theory, and mean-field results.

The aspect of screening of the intramolecular excluded volume shall be neglected in this paper. It would require the use of self-consistent PRISM theory, which is considerably more demanding [10]. Moreover, the errors made, when neglecting the crossover to Gaussian intramolecular correlations on length scales large compared to the density screening length $\xi_{\rho}$, will not affect the scalings of the intermolecular correlations for distances smaller than $\xi_{\rho}$, which are the main focus of this paper. Thus, in the following the intramolecular correlations shall be characterized by a density-independent polymer structure factor $\omega_{q}$, which, for macromolecules of $N$ segments at the positions $\mathbf{r}_{\alpha}$, is defined as follows:

$$
\omega_{q}=\frac{1}{N} \sum_{\alpha, \beta=1}^{N}\left\langle e^{i \mathbf{q} \cdot\left(\mathbf{r}_{\alpha}-\mathbf{r}_{\beta}\right)}\right\rangle
$$

Its full functional form will not be required. Knowledge of its variation for small, large, and intermediate wave vectors suffices $[1,2]$.

For small wave vectors, the number of scattering units, the index of polymerization $N$, where $N \gg 1$ for macromolecules, and the global molecular size, the radius of gyration $R_{g}$, can be obtained from a scattering experiment measuring $\omega_{q}$ : 


$$
\omega_{q} \rightarrow N\left(1-\frac{1}{d} q^{2} R_{g}^{2}+\cdots\right) \text { for } q R_{g} \ll 1
$$

where $d$ is the spatial dimension. In an intermediate wavevector range, the macromolecule is supposed to be selfsimilar. This leads to a power-law behavior in $\omega_{q}$ determined by the fractal dimension, $d_{F}=1 / \nu$ :

$$
\omega_{q} \rightarrow \frac{1}{(q \sigma)^{1 / \nu}} \text { for } 1 / R_{g} \ll q \ll 1 / \sigma \text {. }
$$

The fractal exponent $\nu$ also determines the size-mass scaling, $R_{g} \propto \sigma N^{\nu}$, where a smooth crossover from Eq. (2) to Eq. (3) is assumed around $q R_{g} \approx 1$. The assumption of an intermediate self-similar molecular structure rules out the study of compact macromolecules, e.g., hard-spherelike colloids, but is appropriate for polymer chains in good, $\nu=0.588 \ldots$, or $\Theta$ solvents, $\nu=\frac{1}{2}$, or for rods $\nu=1$, which share some properties with semiflexible polymer molecules like actin or DNA $[2,1,18]$. The Kuhn'sche-segment size $\sigma$ in Eq. (3) is of the order of local polymer-specific length scales where microscopic segmental packing effects influence the complicated structure of $\omega_{q}$. This chemistry-dependent variation of $\omega_{q}$ around $q \sigma \approx 1$ can be included in PRISM studies [10], but shall be neglected here. Only the self-scattering contribution, $\alpha=\beta$ in Eq. (1), which is the only remaining contribution for large wave vectors, $q \sigma \gg 1$, is universal and needs to be considered:

$$
\omega_{q} \rightarrow 1 \quad \text { for } q \sigma \gg 1
$$

Thus, a generic smooth crossover from the point particle self-scattering term, Eq. (4), to the self similar intramolecular correlations, Eq. (3), will be assumed. Chemistry-dependent local packing will show up in all correlation functions on microscopic length scales but will not, except for in prefactors, affect the intermolecular structure on global length scales like the molecule size $R_{g}$ or the mesh width $\xi_{\rho}$, as will be shown explicitly.

In order to characterize the total, including the intermolecular, density correlations of an interacting polymer system, further correlation functions need to be introduced. In order to compare them with results from other approaches it is useful to recall their definition as used in PRISM theory $[10,19]$. To be specific, let us consider $n$ polymers with $N$ scattering units in a $d$-dimensional volume $V$, where in the thermodynamic limit the number density of segments, $\varrho$ $=n N / V$, is kept fixed, i.e., $n, V \rightarrow \infty$ with $\varrho=$ const. The local, fluctuating density is

$$
\varrho(\mathbf{r}, t)=\sum_{i=1}^{n} \sum_{\alpha=1}^{N} \delta\left(\mathbf{r}-\mathbf{r}_{\alpha}^{(i)}(t)\right)
$$

with equilibrium average $\langle\varrho(\mathbf{r}, t)\rangle=\varrho$. The spatial components of the density fluctuations shall be denoted by $\varrho_{\mathbf{q}}$, where

$$
\varrho_{\mathbf{q}}=\int d^{d} r e^{i \mathbf{q} \cdot \mathbf{r}} \varrho(\mathbf{r})=\sum_{i=1}^{n} \sum_{\alpha=1}^{N} e^{i \mathbf{q} \cdot \mathbf{r}_{\alpha}^{(i)}}
$$

Their statistical average vanishes except for zero wave vector, $\left\langle\varrho_{\mathbf{q}}\right\rangle=\varrho \delta_{\mathbf{q}, \mathbf{0}}$.

The total structure factor $S_{q}$ as measured, for example, by coherent neutron scattering $[2,19]$ is given by the second moment of the wave-vector-dependent density fluctuations, from Eq. (6):

$$
\begin{aligned}
S_{q} & =\frac{1}{n N}\left\langle\varrho_{q}^{*} \varrho_{q}\right\rangle-n N \delta_{\mathbf{q}, \mathbf{0}} \\
& =\frac{1}{n N} \sum_{i, j=1}^{n} \sum_{\alpha, \beta=1}^{N}\left\langle e^{i \mathbf{q} \cdot\left(\mathbf{r}_{\alpha}^{(i)}-\mathbf{r}_{\beta}^{(j)}\right)}\right\rangle-n N \delta_{\mathbf{q}, \mathbf{0}} .
\end{aligned}
$$

The total density fluctuations are straightforwardly separated into density fluctuations on the identical polymer $\omega_{q}$, the intramolecular structure factor, and on different polymers, $h_{q}$, the intermolecular structure factor.

$$
S_{q}=\omega_{q}+\varrho h_{q},
$$

where the intramolecular part has already been defined in Eq. (1) above. The intermolecular structure factor $h_{q}$ describes the packing of different molecules and is given by the restricted $\operatorname{sum} i \neq j$ :

$$
h_{q}=\frac{V}{n^{2} N^{2}} \sum_{i, j=1, i \neq j}^{n} \sum_{\alpha, \beta=1}^{N}\left\langle e^{i \mathbf{q} \cdot\left(\mathbf{r}_{\alpha}^{(i)}-\mathbf{r}_{\beta}^{(j)}\right)}\right\rangle-V \delta_{\mathbf{q}, \mathbf{0}} .
$$

The intermolecular structure factor is the Fourier transform of the intermolecular pair correlation function $g(r)$ :

$$
h_{q}=\int d^{d} r e^{i \mathbf{q} \cdot \mathbf{r}}[g(r)-1]
$$

The pair-correlation function describes the probability averaged over all segments of finding at a distance $r$ from site $\alpha$ on molecule $i$, another segment $\beta$ of a different molecule $j$. From Eqs. (9) and (10), one obtains

$$
g(r)=\frac{V}{n^{2} N^{2}} \sum_{i, j=1 ; i \neq j} \sum_{\alpha, \beta=1}^{N}\left\langle\delta\left(\mathbf{r}-\left(\mathbf{r}_{\alpha}^{(i)}-\mathbf{r}_{\beta}^{(j)}\right)\right)\right\rangle .
$$

$g(r)$ is non-negative and approaches unity for large separations $r$, because then statistical correlations between the sites at $\mathbf{r}_{\alpha}^{(i)}$ and $\mathbf{r}_{\beta}^{(j)}$ have vanished [19].

Implicit in the Eqs. (7)-(11) is a neglect of a special site dependence of the density fluctuations as it might, for example, arise from chain-end effects for linear polymers [9]. Star polymers, where sites in the core region possibly experience very different local-density fluctuations than sites in the star arms, also would require a more elaborate treatment [10]. Nevertheless, for arbitrary macromolecular architectures, the above-defined correlation functions are experimentally measurable, at least in principle, and can also be determined from computer simulations. They contain information about the local liquid structure and remain meaningful in the whole accessible density range, from dilute solutions to melts. 


\section{PRISM INTEGRAL EQUATIONS}

Whereas the effects of the excluded volume on the intramolecular structure, like swelling, are already taken into account in Eq. (1), PRISM theory $[9,10]$ explicitly enforces intermolecular excluded volume by requiring the pair correlation function to vanish for distances smaller than the segment size $\mathbf{R}$.

$$
g(r)=0 \text { for } r<R
$$

Building upon the Ornstein-Zernicke approach so successful for simple liquids [19], an averaged molecular site-site Ornstein-Zernicke-like equation $[11,12]$ is formulated and can be viewed as definition of an effective potential, the direct correlation function $c_{q}$ :

$$
h_{q}=\omega_{q} c_{q}\left(\omega_{q}+\varrho h_{q}\right) .
$$

Equations (8) and (13) can also be brought into a RPA-like form, which supports the interpretation of the direct correlation function as an effective potential.

$$
S_{q}^{-1}=\omega_{q}^{-1}-\varrho c_{q} .
$$

Different from the RPA approach, $c_{q}$ is not considered to be given, but needs to be found from a solution of the nonlinear integral equations. Besides Eqs. (12)-(14), a further equation, the "closure" approximation, is required to determine the solution. The most simple and yet appropriate closure to treat the intermolecular steric repulsion is the Percus-Yevick (PY) approximation, which expresses the expectation that the effective potential is short-ranged:

$$
c(r)=0 \text { for } r>R \text {. }
$$

Note that the interaction described by $c(r)$ is localized on microscopic length scales. Thus, the PRISM equations describe the interplay of local intermolecular steric interactions and long-ranged intramolecular correlations due to macromolecular connectivity $[9,10]$.

Thread limit for (semi-) dilute solutions. In the simplified non-self-consistent PRISM approach, the intramolecular structure $\omega_{q}$, from Eq. (1), is assumed to be given, and (mostly numerical) techniques to solve the integral equations, Eqs. (12) -(15) are employed [10]. Note that for a Pade approximation to $\omega_{q}$ for Gaussian chains with $\nu=\frac{1}{2}$ in $d$ $=3$ an analytic solution of the PRISM equations on all length scales exists [16]. The solution technique employing the Wiener-Hopf factorization as pioneered by Baxter [20] can straightforwardly be extended to Gaussian chains in (low) odd dimensions, $d=5,7, \ldots$, but a simpler approach can also be used in order to study the low-density results of PRISM theory analytically. For the mentioned case, $\nu=\frac{1}{2}$ in $d=3$, this was first used in $[13,14]$, explicitly justified in [16], and without proof extended to exponents $\nu$ within the bounds $1 / d \leqslant \nu<2 / d$ in [15]. Here, general arguments on the solutions of Eqs. (12)-(15) allow us to find the low-density limits in the more general case $1 / d<\nu$ and $d \geqslant 2$.

The special low-density limit, called the "thread PRISM" model $[13,14]$, studied in the following assumes that polymer solutions can be modeled as a low-density limit of the one-component PRISM equations for polymer melts. Special solvent effects are assumed to be taken into account via the model for the intramolecular structure, Eqs. (2)-(4).

In general, the excluded volume constraint, Eq. (12), and the PY closure, Eq. (15), lead to a discontinuity in the paircorrelation function $g(r)$ and in the direct-correlation function $c(r)$ at contact:

$$
\begin{gathered}
g_{d}:=g(r \searrow R)>0, \\
c(R-):=c(r \nearrow R) \neq 0 .
\end{gathered}
$$

Note that the actual values of $g_{d}$ and $c(R-)$ will be dependent on details of the monomer chemistry, as the segment size $R$ obviously is a microscopic length. To connect both quantities it is useful to express $h_{q}$ and $c_{q}$ as onedimensional Fourier transforms,

$$
\begin{aligned}
& h_{q}=\int d r e^{i q r} j(r), \\
& c_{q}=\int d r e^{i q r} i(r),
\end{aligned}
$$

with the symmetric functions,

$$
\begin{gathered}
j(r)=\Omega_{d-1} \int_{|r|}^{\infty} d s s(g(s)-1)\left(s^{2}-r^{2}\right)^{(d-3) / 2}, \\
i(r)=\Theta(R-|r|) \Omega_{d-1} \int_{|r|}^{R} d s s c(s)\left(s^{2}-r^{2}\right)^{(d-3) / 2},
\end{gathered}
$$

where $\Omega_{d}=2 \pi^{d / 2} / \Gamma(d / 2)$ denotes the surface of the $d$-dimensional unit sphere. Because of Eqs. (12) and (15), $i(r)$ can be nonsmooth for $|r| \leqslant R$ only, while this can happen for $j(r)$ for $|r| \geqslant R$. Thus, using the large wave-vector limits, $h_{q} \propto-g_{d} \cos q R / q^{d-1}$ for $q \rightarrow \infty$ and similarly for $c_{q}$, and the large wave-vector asymptote of $\omega_{q}$, see Eq. (4), Eq. (13) connects the discontinuities of $g(r)$ and $c(r)$ at $r=R$, leading to

$$
g_{d}=-c(R-) .
$$

Moreover, one concludes that $c(r)$ is finite. Thus, the scaling of the Fourier transform of the direct-correlation function with global parameters (to be defined below) also can be connected to the contact value:

$$
c_{q}=R^{d} c(R-) f_{c}(q R)=:-g_{d} R^{d} f_{c}(q R),
$$

where the regular function, $f_{c}(x)=\int_{y<1} d^{d} y e^{i \mathbf{x} \cdot \mathbf{y}} c(R \mathbf{y}) /$ $c(R)$, has a finite value at $x=0$. For the analytically known results of the PRISM equations these properties could be shown explicitly [16], and they can now be used to simplify the PRISM equation for low densities.

In order to extract the large distance solution of the PRISM equations, it proves useful to shift the microscopic length scales to zero:

$$
R \rightarrow 0, \quad \sigma \rightarrow 0 \quad \text { with } \quad \sigma / R=\text { const. }
$$

In order to evade the trivial limit of a noninteracting ideal gas, the length scale of the intramolecular correlations, to be 
denoted by $\xi_{c}$, which is proportional to the molecular size $\xi_{c} \propto R_{g}$, is kept finite by increasing the index of polymerization $N$.

$$
N \rightarrow \infty \text { so that } \xi_{c} \propto \sigma N^{\nu}=\text { const. }
$$

Also, in order to keep intermolecular excluded volume active, the bare segmental density is increased beyond bounds:

$$
\varrho \rightarrow \infty \text { so that } \varrho / \varrho_{*}=\text { const. }
$$

In the thread PRISM equations, there enters a typical density, the dilute to semidilute crossover density $\varrho_{*}$, which is familiar from scaling considerations [1]. As will be shown below, $\varrho_{*}$ is defined differently for scaling exponents $\nu$ below and above a value $\nu_{c}$, which denotes the crossover to meanfield-like behavior:

$$
\varrho_{*} \propto \begin{cases}\frac{N}{\xi_{c}^{d}} & \text { for } \quad \nu<\nu_{c}=\frac{2}{d} \\ \frac{1}{N \sigma^{d}} & \text { for } \quad \nu>\nu_{c} .\end{cases}
$$

Equations (21)-(24) specify the thread PRISM limit. Note that the divergent number density $\varrho_{*}$ actually corresponds to a vanishing polymer volume fraction $\phi$ and thus (semi-) dilute polymer solutions are studied as claimed:

$$
\phi_{*}=\varrho_{*} \sigma^{d} \propto \begin{cases}1 / N^{(\nu d-1)} & \text { for } \quad \nu<\nu_{c} \\ 1 / N & \text { for } \quad \nu>\nu_{c},\end{cases}
$$

where $\phi=\mathcal{O}(1)$ corresponds to polymer melts.

The solution of the nonlinear PRISM integral equations in the general case, of course, demands to find $f_{c}(x)$ from Eq. (20) for all $x$. However, a solution to Eqs. (12)-(15) depending on $f_{c}(0)$ can be constructed on large distances $r \gg R, \sigma$, or, equivalently in the thread limit, for finite distances, $r$ $>0$. This holds, because in the limit of $R \rightarrow 0$, the excluded volume condition Eq. (12) affects a point (of measure zero) only, and thus $h_{q}$ can be obtained from the Fourier integral of $g(r)$ outside the core $r>0$. From the inverse transformation and Eqs. (13) and (20), the contact value follows in the general case where $\mathbf{R}$ denotes a vector of length $R+$ :

$$
g_{d}-1=-\frac{N A}{\varrho} \int \frac{d^{d} q}{(2 \pi)^{d}} e^{i \mathbf{q} \cdot \mathbf{R}} \frac{\bar{f}_{c}(q R) \bar{\omega}_{q}^{2}}{1+A \bar{f}_{c}(q R) \bar{\omega}_{q}} .
$$

The normalized functions $\bar{\omega}_{q}=\omega_{q} / N$ and $\bar{f}_{c}(x)$ $=f_{c}(x) / f_{c}(0)$ have been introduced, and the long wavelength interaction parameter $A$, which abbreviates the zero wave-vector limit of the direct-correlation function:

$$
A=-N \varrho c_{q=0}=N \varrho g_{d} R^{d} f_{c}(0) .
$$

For (semi-) dilute solutions, the limit Eq. (21) simplifies the PRISM integral equations, because the core condition, Eq. (12), becomes irrelevant, and Eq. (26) assures that the connection Eq. (19) is satisfied. The two further conditions, Eqs. (22) and (23), assure that nontrivial solutions describing an interacting polymer solution are obtained. Because of Eq. (22), only the long-ranged scaling form of the intramolecular structure factor enters:

$$
\bar{\omega}_{q}=\bar{\omega}\left(x=q \xi_{c}\right)= \begin{cases}1+O\left(x^{2}\right) & x \rightarrow 0 \\ 1 / x^{1 / \nu} & x \rightarrow \infty\end{cases}
$$

And Eq. (23) enforces the molecules to interact, $A \neq 0$, so that Eq. (26) leads to a transcendental equation determining $A$ (equivalently $g_{d}$ ), which is the only unknown parameter in the thread intermolecular structure factor:

$$
\varrho h_{q}=-N A \frac{\bar{\omega}_{q}^{2}}{1+A \bar{\omega}_{q}} \text {. }
$$

\section{THREAD LIMIT RESULTS}

In the dilute to semidilute concentration region, the thread PRISM result for $h_{q}$ adopts the RPA-like form, Eq. (29), where the interaction parameter $A$ needs to be found from Eq. (26). The total structure factor then also has simple RPAlike form:

$$
S_{q}=N \frac{\bar{\omega}_{q}}{1+A \bar{\omega}_{q}},
$$

where generally $\bar{\omega}_{q}$ differs from the Gaussian form and the integrated intermolecular interaction strength, $A$, in general, differs from the simple RPA approximation, $A \propto N \varrho R^{d}$. Because of the large wave-vector behavior of $\bar{\omega}$, Eq. (28), the limit $R \rightarrow 0$ affects the integral in Eq. (26) differently for $\nu$ $\left\langle\nu_{c}=2 / d\right.$ or $\nu>\nu_{c}$. In the first case, the integral converges uniformly, and integration and limit can be interchanged, thus only $\bar{f}_{c}(0)=1$ enters. In the second case, the integral converges only because of the wave-vector dependence of $\bar{f}_{c}(q R)$, and leads to RPA or mean-field behavior.

\section{A. Below the mean-field crossover}

In the thread equation for the interaction parameter $A$, Eq. (26), the limit $R \rightarrow 0$ can be performed trivially for $\nu<\nu_{c}$ $=2 / d$, and $A$ becomes a function of $\varrho / \varrho_{*}$ only, where the crossover density $\varrho_{*}=N \Omega_{d} /\left(2 \pi \xi_{c}\right)^{d}$ enters:

$$
\left(1-g_{d}\right) \frac{\varrho / \varrho_{*}}{A}=\int_{0}^{\infty} d x \frac{x^{d-1} \bar{\omega}^{2}(x)}{1+A \bar{\omega}(x)} .
$$

For size-mass scaling exponents $\nu$ corresponding to fractal dimensions, $d_{F}=1 / \nu$, equal to or exceeding the spatial dimension, i.e., for $\nu<1 / d$, the intramolecular structure on long length scales determines the thread Eq. (31). There, screening of the intramolecular excluded volume has been neglected in the present approach, and the polymer segregation effect predicted in the thread limit requires use of the self-consistent PRISM approach [15]. In order to avoid this complication, the exponent $\nu$ will be restricted in the following, $\nu>1 / d$. From the two limits of the integral in Eq. (31), constant for $A \ll 1$ and $A^{-(2-\nu d)}$ for $A \gg 1$, the scaling form for the thread parameter can be determined:

$$
A=\left(\varrho / \varrho_{*}\right) f_{A}\left(\varrho / \varrho_{*}\right)
$$




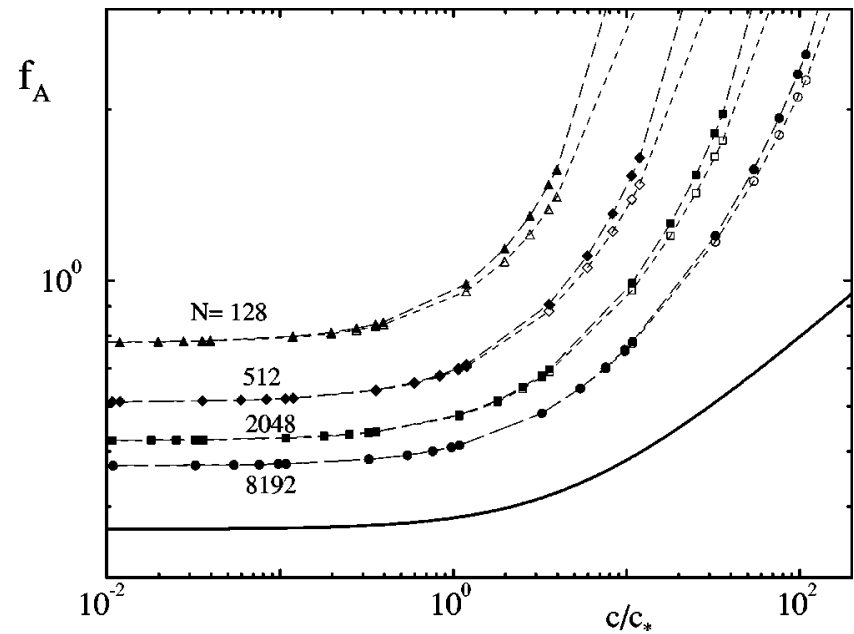

FIG. 1. Scaling function $f_{A}\left(c / c_{*}\right)$ (bold solid curve) of the thread interaction parameter $A$ versus rescaled (molecular) polymer concentration in double logarithmic presentation for the Flory exponent, $1 / \nu=1.67 ; c_{*}=1 /\left(2 \pi^{2} \xi_{c}^{3}\right)$ is the molecular overlap concentration. The curves labeled with degree of polymerization $N$ are results from microscopic PRISM calculations of the model described in the text, where $\xi_{c}=0.28 N^{\nu} \bar{\sigma}$ is found with Eq. (28). Full symbols give $f_{A}$ determined from $S_{q \rightarrow 0}$, and open symbols from the contact value $g_{d}$ shifted by model-dependent factors $(0.088$, $0.086,0.085$, and 0.085 with increasing $N$ ).

where

$$
f_{A}(x) \propto\left\{\begin{array}{lll}
\text { const }+O(x) & \text { for } & x \rightarrow 0 \\
x^{(2-\nu d) /(\nu d-1)} & \text { for } & x \rightarrow \infty
\end{array}\right.
$$

In Eq. (32), the contact value correction was already neglected, as it is of higher order, as can be deduced from Eq. (27). Actually, a scaling law follows from Eqs. (27) and (32) for the contact value:

$$
g_{d}=c \frac{\left(\xi_{c} / R\right)^{d}}{N^{2}} f_{A}\left(\varrho / \varrho_{*}\right),
$$

where the identical scaling function $f_{A}$ from Eq. (32) enters. The numerical prefactor $c$, of course, depends on the microscopic details of the polymer model. Note that for $\nu<\nu_{c}$ the contact value vanishes like $N^{-(2-\nu d)}$ for $N \rightarrow \infty$ in the dilute case. The scaling function $f_{A}$ also determines the density dependence of the mesh size or density screening length; as for large reduced densities, $\varrho \gg \varrho_{*}$, the width of the total structure factor can be estimated from $S_{q}=(N / A) /(1$ $\left.+\left(q \xi_{\rho}\right)^{1 / \nu}\right)$, for $q \xi_{c} \gg 1$, which leads to

$$
\xi_{\rho}=c^{\prime} \sigma\left(\varrho \sigma^{d}\right)^{-\nu /(\nu d-1)} .
$$

For a given model of the intramolecular structure factor, the thread equation, Eq. (31) with $g_{d}=0$, allows one to determine $f_{A}$ straightforwardly. Figure 1 shows the result for the polymer model: $\omega_{q}=(1 / N) \sum_{\alpha, \beta=1}^{N} \exp -\left(q^{2} \bar{\sigma}^{2} / 6\right)|\alpha-\beta|^{2 \nu}$, with the exponent given by the Flory approximation $\nu=3 / 5$ corresponding to good polymer solutions $[1,2]$. Numerical solutions of the microscopic PRISM Eqs. (12)-(15) for this model and for not too large degrees of polymerization $N$, still exhibit rather large corrections to the thread asymptote. This can be expected to be model dependent. From Fig. 1 one notices that the connection of the contact value to the small wave-vector interaction parameter, Eq. (27), already holds for values of $N$ where the asymptotic $f_{A}$, Eq. (32), is not yet reached. Differences appear for larger packing fractions and indicate concentrated or meltlike polymer packing.

The pair-correlation function $g(r)$ in the thread limit can be obtained for finite segment distances from the Fourier transform of Eq. (29). For dilute densities, $\varrho \ll \varrho_{*}$ and thus $A \ll 1$, it differs from the ideal gas limit, $g(r)=1$, because of two molecule interactions. In the semidilute concentration region, $\varrho \gg \varrho_{*}$ and $A \gg 1$, the replacement $A=\left(\xi_{c} / \xi_{\rho}\right)^{1 / \nu}$ shows that $g(r)$ depends on the two length scales, $\xi_{c}$ and $\xi_{\rho}$, independently. On length scales large compared to the mesh size $r \gg \xi_{\rho}$, the intermolecular structure factor exhibits the well-known correlation hole $[1,9,10,15]$, which asymptotically for $\varrho \gg Q_{*}$ exactly cancels off the long-ranged intramolecular correlations:

$$
h_{q} \rightarrow-\frac{N}{\varrho} \bar{\omega}\left(q \xi_{c}\right) \quad \text { for } \quad q \ll 1 / \xi_{\rho} ; \varrho \gg \varrho_{*} .
$$

This result is equivalent to $S_{q} \ll \omega_{q}$ for $q \xi_{\rho} \ll 1$ in the semidilute range [1]. Note that the self-similar structure of the molecule leads to the power-law behavior $\varrho h_{q} \propto-(\sigma q)^{-1 / \nu}$ for $1 / \xi_{c} \ll q \ll 1 / \xi_{\rho}$, which is equivalent to a power-law variation in the pair-correlation function: $g(r)-1 \propto(-1 /$ $\varrho)(\sigma / r)^{d-1 / \nu}$ for $\xi_{\rho} \ll r \ll \xi_{c}$ [15]. For larger distances, $r$ $\gg \xi_{c}$, Eq. (35) describes how $g(r)$ decays exponentially to its random mixing value unity.

Within the mesh size, i.e., for distances around and smaller than the density screening length, the intermolecular correlations do not depend on the molecular size and $\bar{\omega}$ in Eq. (29) can be replaced by its large $q \xi_{c}$ asymptote from Eq. (28). This leads to

$$
h_{q} \rightarrow \frac{-\bar{c} \xi_{\rho}^{d}}{\left(q \xi_{\rho}\right)^{1 / \nu}+\left(q \xi_{\rho}\right)^{2 / \nu}} \text { for } 1 / \xi_{c} \ll q ; \varrho \gg \varrho_{*},
$$

where the limiting behaviors of $h_{q}$ for $q \xi_{\rho}$ large or small compared to unity can be read of immediately and $\bar{c}$ $=N /\left(\varrho A \xi_{\rho}^{d}\right)$ approaches a number $(\bar{c} \rightarrow 6.26 \ldots$, for $\nu$ $=3 / 5$ ). Note that Eq. (34) for the density screening length ensures a smooth crossover of Eq. (36) to Eq. (35) in the correlation hole region. The variation of the pair-correlation function, which describes the mesh structure for $r \ll \xi_{c}$, can thus be obtained in closed form, if the neglect of the cutoff of the correlation hole at $r \gtrsim \xi_{c}$ included in Eq. (35), is kept in mind. For $r \ll \xi_{c}, g(r)$ depends on $r / \xi_{\rho}$ only, with

$$
\begin{aligned}
g(r) & =1-\bar{c} \int \frac{d^{d} y}{(2 \pi)^{d}} e^{-i \mathbf{y} \cdot \mathbf{r} / \xi_{\rho}} \frac{1}{y^{1 / \nu}+y^{2 / \nu}} \\
& \rightarrow\left\{\begin{array}{lll}
\left(r / \xi_{\rho}\right)^{2 / \nu-d}, & r \ll \xi_{\rho}, & r \gg \sigma, R \\
1-\left(\xi_{\rho} / r\right)^{d-1 / \nu}, & r \gg \xi_{\rho} ; & r \ll \xi_{c},
\end{array}\right.
\end{aligned}
$$

where $\bar{c}$ ensures $g(0)=0$ in agreement with Eq. (31) and constant prefactors of order unity have been suppressed in the final two lines. For polymer chains in good solvents, the smooth increase, $g\left(r \ll \xi_{\rho}\right) \propto\left(r / \xi_{\rho}\right)^{1 / 3}$, by accident agrees with the estimate from Ref. [21], $g\left(r \ll \xi_{\rho}\right) \propto\left(r / \xi_{\rho}\right)^{(\gamma-1) / \nu}$ 
$\approx\left(r / \xi_{\rho}\right)^{1 / 3}$, where $\gamma$ is associated with the entropy of a single polymer chain [2]. The depth of the correlation hole displays an intriguing dependence on the fractal and spatial dimensionalities. The probability to find a segment of another polymer within the considered molecule decreases strongly if $1 / \nu \rightarrow d$. From Eq. (37) one estimates $g\left(r \approx \xi_{c}\right)-1 \propto$ $-1 / N^{\nu d-1}$, which becomes a number of order unity in the case $\nu=1 / d$. The smooth variation of $g(r)$ at short distances explains why the scaling of the correct contact value $g_{d}$, Eq. (33), with macroscopic variables can be estimated from the thread solution, Eq. (37), by $g_{d} \propto g(\sigma)$; its dependence on the ratio of the microscopic length scales $\sigma / R$, however, cannot generally be recovered in this way [16]. Note that Eqs. (34)(37) asymptotically apply for semidilute solutions, $\varrho \gg \varrho_{*}$, whereas Eqs. (29), (30), and (32) describe the full dilute-tosemidilute crossover region.

\section{B. The mean-field cases}

The condition Eq. (26) for the contact value $g_{d}$, or equivalently, for the thread parameter $A$ becomes independent of the microscopic interaction details only for $\nu<\nu_{c}$. Above the crossover exponent, $\nu>\nu_{c}=2 / d$, the integral over the effective potential as it enters $h_{q}$ is determined by the local structure in $f_{c}(q R)$. In the thread limit, Eq. (26) becomes a linear, density-independent equation for $g_{d}$ with solution:

$$
g_{d}=1 /\left[1+\int \frac{d^{d} q}{(2 \pi / R)^{d}} e^{i \mathbf{q} \cdot \mathbf{R}}(q \sigma)^{-2 / \nu} f_{c}(q R)\right] .
$$

Thus, a finite density-independent contact value follows in the mean-fieldlike cases $\nu>\nu_{c}$. Obviously, its exact value depends on the solution of the PRISM equations considering all microscopic details and is beyond the reach of the thread PRISM approach. The interaction parameter $A$ thus shows the density scaling as expected within RPA. The reduced density $\varrho / \varrho_{*}$ appears, with $\varrho_{*}=1 /\left(N \sigma^{d}\right)$, and $A$ becomes, with an unknown numerical constant $\widetilde{c}$, which, however, may depend on the ratio $\sigma / R$ of the microscopic length scales:

$$
A=\widetilde{c} \varrho / \varrho_{*} .
$$

In the semidilute density regime, the width of the total structure factor again determines the density screening length, $S_{q}=(N / A) /\left(1+\left(q \xi_{\rho}\right)^{1 / \nu}\right)$ for $q \xi_{c} \gg 1$, with the result

$$
\xi_{\rho}=\widetilde{c}^{\prime} \sigma\left(\varrho \sigma^{d}\right)^{-\nu} .
$$

Again, the numerical prefactor $\widetilde{c}^{\prime}$ depends on the polymer model. The result, Eq. (35), discussed for the intermolecular structure in the correlation hole region holds. The mesh structure factor $h\left(q \gg 1 / \xi_{c}\right)$, however, shows a different density scaling:

$$
h_{q} \rightarrow \frac{-\hat{c} \sigma^{d}\left(\xi_{\rho} / \sigma\right)^{2 / \nu}}{\left(q \xi_{\rho}\right)^{1 / \nu}+\left(q \xi_{\rho}\right)^{2 / \nu}} \text { for } 1 / \xi_{c} \ll q
$$

that again, with Eq. (40), leads to a smooth crossover for intermediate distances, $1 / \xi_{c} \ll q \ll 1 / \xi_{\rho}$, but to a small distance divergence of the thread pair-correlation function, $g(r) \propto-(\sigma / r)^{(d-2 / \nu)}$ for $r \ll \xi_{\rho}$. This results from the neglect of the wave-vector variation of the direct-correlation function, Eq. (20), and reinforces that the validity of the thread $g(r)$ is restricted to $r \gg \sigma$ for $\nu>\nu_{c}$, where $g(r)$ is still positive, as it must be by definition.

\section{DISCUSSION AND COMPARISON WITH OTHER APPROACHES}

In this paper, scaling limits appropriate for the dilute to semidilute concentration regime of macromolecular solutions have been derived starting from the microscopic PRISM integral equations. RPA-like expressions for the total density fluctuations, the structure factor $S_{q}$, Eq. (30), were given, where the density scaling of the interaction parameter $A$ was deduced from the local excluded volume constraint, Eq. (12). The thread interaction parameter is connected to the more familiar excluded volume parameter $\mathrm{v}$ via $A$ $=\left(N_{A} M \varrho / M_{0}^{2}\right) \mathrm{v}$, where $N_{A}$ is Avogadro's number, $M$ is the molecular weight, and $M_{0}$ is the monomer weight. Effective density-dependent excluded volume parameters $\mathrm{v}(\varrho)$ have often been used in connection with RPA expressions [3,21], and Eq. (32) justifies this.

The crossover of the single chain correlations to Gaussian large-distance behavior for $r \gg \xi_{\rho}$ due to intramolecular excluded volume has been neglected and would affect the model for $\omega_{q}$, Eq. (5), and, consequently, the thread results for large distances. Use of self-consistent PRISM [10] to incorporate this would be required, but Eq. (32) for the thread parameter $A$ indicates that no change of its density scaling can be expected.

The crossover density $\varrho_{*}$, Eq. (24), arises from the full microscopic PRISM equations as the relevant low-density scale, and importantly, the qualitatively different definitions in the mean field, $\nu>\nu_{c}=2 / d$, and in the nontrivial cases, $\nu<\nu_{c}$, are recovered. Whereas for $\nu<\nu_{c}$ the molecular crossover density, $c_{*}=\varrho_{*} / N$, is defined in terms of the molecular size only, $c_{*} \propto 1 / R_{g}^{d}$ for $\nu>\nu_{c}$; in the mean-field cases also a microscopic length, the segmental hard-core diameter $R$, enters $c_{*} \propto 1 /\left(R_{g}^{2 / \nu} R^{d-2 / \nu}\right)$ for $\nu>\nu_{c}$. This indicates that intermolecular steric interactions become important as soon as the macromolecules fill space for $\nu<\nu_{c}$, whereas for the more open molecules, $\nu>\nu_{c}$, much higher densities are required.

For chain polymers, the upper critical dimension, which separates mean-field and fluctuation-dominated structures, agrees with the renormalization-group results, $d_{c}=2 / \nu_{c}=4$ $[1,2]$. For rod polymers, $\nu=1>\nu_{c}$, the mean-field-like behavior underlies the successful Onsager theory of the nematic transition [22] and is generally argued to be true [23]. Note that in the studied PRISM theory orientational, "nematic" interactions are not treated correctly [10], and thus a nematic transition for rods is missed. Very recently, PRISM has been generalized to treat oriented polymer fluids and the isotropic-nematic liquid crystal transition [24]. As the (isotropic) crossover density $c_{*}$ for rods is of the order of the nematic transition density [22], a suppression of nematic order is required to study the described isotropic semidilute rod solutions experimentally. Networks of stiff semiflexible molecules like actin may provide good systems [18].

Of course, the full PRISM integral equations, which have 
been introduced to study dense polymer systems with shortranged meltlike correlations [9], incorporate wave-vectordependent corrections in, e.g., the effective interaction $c_{q}$, see Eq. (14), when $1 / q$ approaches local length scales.

From the compressibility, which is connected to the zero wave-vector limit of the total structure factor, the equation of state can be obtained, where $\Pi$ denotes the osmotic pressure $[19,10]$ :

$$
\begin{aligned}
& \frac{\Pi}{\varrho k_{B} T}=\frac{1}{N}+\frac{1}{\varrho} \int_{0}^{\varrho} d \varrho^{\prime} \frac{A\left(\varrho^{\prime}\right)}{N} \\
& \propto \begin{cases}\frac{1}{N}\left[1+\mathcal{O}\left(\varrho / \varrho_{*}\right)\right], & \varrho \ll \varrho_{*} \\
\left(\varrho \sigma^{d}\right)^{1 / \nu d-1}, & \varrho \gg \varrho_{*}, \quad \nu<\nu_{c} \\
\varrho \sigma^{d}, & \varrho \gg \varrho_{*}, \quad \nu>\nu_{c},\end{cases}
\end{aligned}
$$

when $A$ is given by Eq. (32). The non-mean-field behavior for $\nu<\nu_{c}$ for semidilute concentrations [15] agrees with the exact Des Cloizeaux result [2], and the second virial coefficient, $1 /\left(N \varrho_{*}\right) \propto R_{g}^{d} / N^{2}$, recovers the picture of dilute polymer coils interacting like hard spheres of radius $R_{g}$ [17], but it does not vanish for dilute $\Theta$ solvents, i.e., for $\nu=\frac{1}{2}$ in the present approach. PRISM theory apparently correctly captures the leading asymptotic behaviors, the free molecule limit $\Pi=(\varrho / N) k_{B} T$ for $\varrho \ll \varrho_{*}$, and the $N$-independent power law for $\varrho \gg \varrho_{*}$, but the next to leading terms are not described correctly, in general.

The structure of the polymer mesh in semidilute solutions, i.e., the intermolecular structure factor $h_{q}$ on length scales of the order of the density screening length $\xi_{\rho}$ has not been conclusively discussed from first principles calculations. The thread PRISM results for the non-mean-field-like case of polymer chains in good solvents give explicit results, Eqs. (36) and (37), which can be compared to results from other approaches.

\section{A. Comparison with scaling considerations}

Detailed scaling law considerations of $h_{q}$ in the limit $q R_{g} \gg 1$ have been presented in [4] and can be directly compared with Eq. (36). The limit, $h_{q} \propto \xi_{\rho}^{d}$ for $1 / R_{g} \ll q \ll 1 / \xi_{\rho}$ strongly differs from the correlation hole behavior, $\varrho h_{q}=$ $-(q \sigma)^{1 / \nu}$ with $\sigma$ the Kuhn'sche-segment size, predicted by the thread PRISM theory for this wave-vector window. Physically, the long-ranged variation of $h_{q}$ arises from the rearrangement of the polymer mesh around a molecule on distances up to the molecule's size. This adjustment compensates for the excess density due to the considered molecule, leading to the small total density fluctuations expected for concentrated systems. The correlation hole has been predicted and discussed for polymer melts [1], but PRISM theory also predicts it for semidilute solutions [9], in agreement with scaling considerations in [21] but in disagreement with the mentioned scaling picture presented in [4]. Intriguingly, PRISM theory, Eq. (37), recovers the tendency of macromolecules to segregate for $\nu=1 / d$ as discussed for ideal chains in two dimensions [1].
In agreement with the thread PRISM result, a scaling law is postulated in Ref. [4] for the intermolecular structure factor inside the coil radius, $h\left(q \gg 1 / R_{g}\right)=\bar{h}\left(q \xi_{\rho}\right)$, which leads to the prediction $h_{q} \propto q^{1-d}$ for $q \rightarrow \infty$ [4]. As a scaling law can only hold for distances large compared to the microscopic length scales, $q \sigma \ll 1$, this result can be compared with the thread scaling power law, $h_{q} \propto q^{-2 / \nu} \xi_{\rho}^{d-2 / \nu}$ in Eq. (36), and again differs. Within thread PRISM the behavior of $h_{q}$ arises naturally as it matches smoothly to the microscopic limit, $h_{q} \sim g_{d} q^{-d} \cos q R$ for $q R \gg 1$, because the contact value vanishes asymptotically, $g_{d} \propto\left(\sigma / \xi_{\rho}\right)^{2 / \nu-d}$ for $\xi_{\rho} \gg \sigma$. This supports the expectation in [21]. Computer simulations could address this question for polymer chain solutions as shown in Sec. IV C, where corrections to the low-density scaling law, Eq. (33), need to be considered, which will arise due to finite packing fractions.

Accepting the existence of a scaling law for the contact value of macromolecules in solutions, Eq. (33) can be used to connect the PRISM results to field-theoretic calculations for two-polymer systems.

\section{B. Comparison with field-theoretic calculations}

Field-theoretic calculations, which employ the mapping of the polymer problem onto the $\mathrm{O}(n \rightarrow 0)$ magnetic model, lead to numerous single-chain results and have recently been extended to provide information about the intermolecular structure factor on short length scales $[4,5]$. In [4], the mentioned behavior $h_{q} \propto q^{-d}$ for $q \rightarrow \infty$ is recovered from the field-theoretic calculation and used to support the scaling picture discussed in the previous section. The implications for $g(r)$ can be compared with another field-theoretic calculation, which studies the number of intersections of two polymer chains [5]. Let $\Sigma_{2}\left(R_{e}\right)$ be the number of intersections of two random (or self-avoiding) walks whose end-toend distance is $R_{e}$ :

$$
\frac{\Sigma_{2}\left(R_{e}\right)}{V\left(4 \pi \sigma^{2}\right)^{d / 2}}=\sum_{\alpha, \beta=1}^{N}\left\langle\delta\left(\mathbf{r}_{0}^{(2)}-\mathbf{r}_{0}^{(1)}-\mathbf{R}_{e}\right) \delta\left(\mathbf{r}_{\alpha}^{(2)}-\mathbf{r}_{\beta}^{(1)}\right)\right\rangle .
$$

For intermediate distances $R_{e}, \sigma \ll R_{e} \ll R_{g}$, where the two polymers overlap but local effects do not dominate $\Sigma_{2}$, the scaling $\Sigma_{2} \propto\left(\sigma / R_{g}\right)^{\omega_{12}(P)}$ is predicted, where the twomolecule correction to scaling exponent $\omega_{12}$ appears [5]. The contact value can now be obtained from $\Sigma_{2}$ by integrating over all possible distances and (trivial) factors of normalization, as can be seen from Eqs. (11), (16), and (21).

$$
g_{d}=\int d^{d} R_{e} \frac{\Sigma_{2}\left(R_{e}\right)}{\left(4 \pi \sigma^{2}\right)^{d / 2} N^{2}} .
$$

Using the results for two polymers from [5] to obtain the scaling of the contact value in the dilute case, one finds

$$
g_{d}^{\mathrm{RG}} \propto \frac{R_{g}^{d-\omega_{12}(P)}}{N^{2}} \text { for } \varrho \rightarrow 0 .
$$

The thread PRISM result, Eq. (32), differs from this, in general, because in PRISM theory the correction to scaling exponent is approximated to $\omega_{12}^{\text {thread }}=0$. Its value in quadratic 


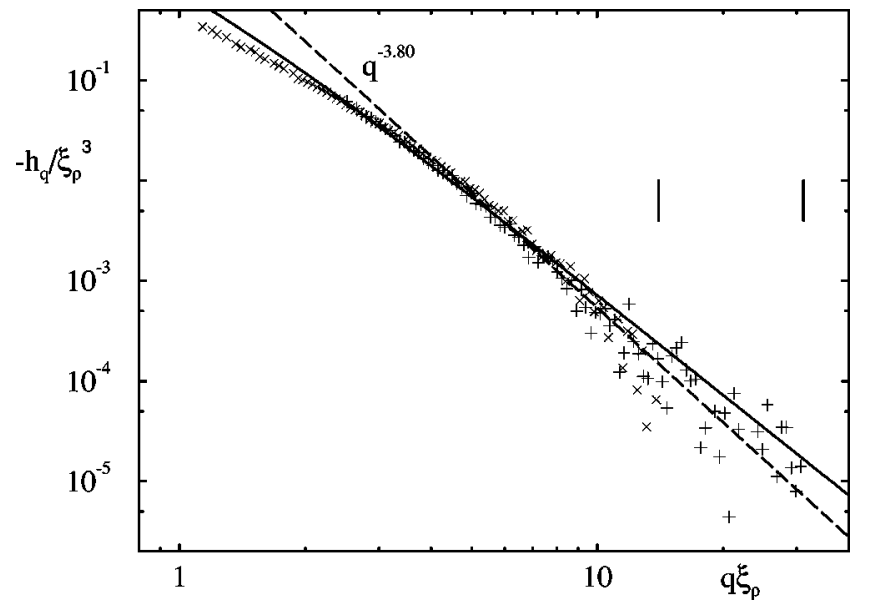

FIG. 2. Intermolecular structure factors $h_{q}$ from a Monte Carlo simulation of the BFM for polymers of length $N=2048$ ( $\xi_{c}=94$ for $\nu=0.588$; all lengths given in units of the lattice constant of the BFM). The data are taken from [27] and are shown scaled with $\xi_{\rho}$ determined from the corresponding $g(r)$ 's of Fig. 3. The +'s belong to the rescaled density $c / c_{*}=25.6$ and the $\times$ 's belong to $c / c_{*}=97.7$. The asymptotic thread PRISM result, Eq. (36), shifted by a correction factor 0.25 is shown as a solid line, whereas the dashed line indicates a power law $q^{-3.80}$ following from Eq. (47). Vertical bars mark where $q=1$.

order in $\varepsilon=4-d$ is known, and the value appropriate for polymer chains in good solvents turns out to be $\omega_{12}(G)$ $=\frac{1}{2} \varepsilon-\frac{19}{64} \varepsilon^{2}+\cdots \approx 0.40$ [5]. It can be compared to the PRISM and to the mean-field approximation, $\omega_{12}^{\mathrm{RPA}}=d$ $-2 / \nu$, which qualitatively differs because it is negative. The thread approximation, $\omega_{12}=0$, is correct at and above the upper critical dimension $d_{c}$.

It appears difficult to envisage simple forms of $g(r)$ that reconcile the prediction $h_{q} \propto q^{-d}$ for $q \rightarrow \infty$ [4] with the results for $\Sigma_{2}$ [5], and the dilute limit of thread PRISM theory qualitatively agrees with the latter.

\section{Comparison with Monte Carlo simulations}

Monte Carlo simulations are well suited to study the intermolecular structure of polymer solutions but face the difficult challenge to achieve a clear separation of the three length scales, segment size $\sigma$ (or excluded volume size $R$ ), density screening length $\xi_{\rho}$, and molecular size $R_{g}$ (or molecular correlation length $\xi_{c}$ ); see the discussion in [6]. Whereas in [6] in the range $1 / R_{g} \ll q \ll 1 / \xi_{\rho}$ a discrimination of the two predictions, $h_{q} \propto$ const. from scaling considerations [4] and $h_{q} \propto q^{-x}$ with $x \approx 1 / \nu$ (as follows from the PRISM treatment of the correlation hole), appears possible and appears to support the latter; no clear conclusions about the exponent in the asymptotic behavior, $h_{q} \rightarrow q^{-x}$ for $1 / \xi_{\rho}$ $\ll q \ll 1 / R$, with $x=d=3$ (scaling picture), $x=4$ (RPA), or $x=2 / \nu \approx 3.34$ (thread PRISM), were possible. Even recent large-scale simulations of the bond fluctuation model (BFM) $[25,26]$ do not provide a conclusive test of the large $q$ dependence if $h_{q}$ is considered [27]. Figure 2 shows data from Ref. [27] for semidilute solutions and rather large chain lengths, $N=2048$, where $\xi_{c}=94, \xi_{\rho}=14$ for $c / c_{*}=97.7$, and $\xi_{\rho}=31.1$ for $c / c_{*}=25.6$, and the steric segment size is $R=2$ in units of the lattice constant of the BFM. For a fit, the

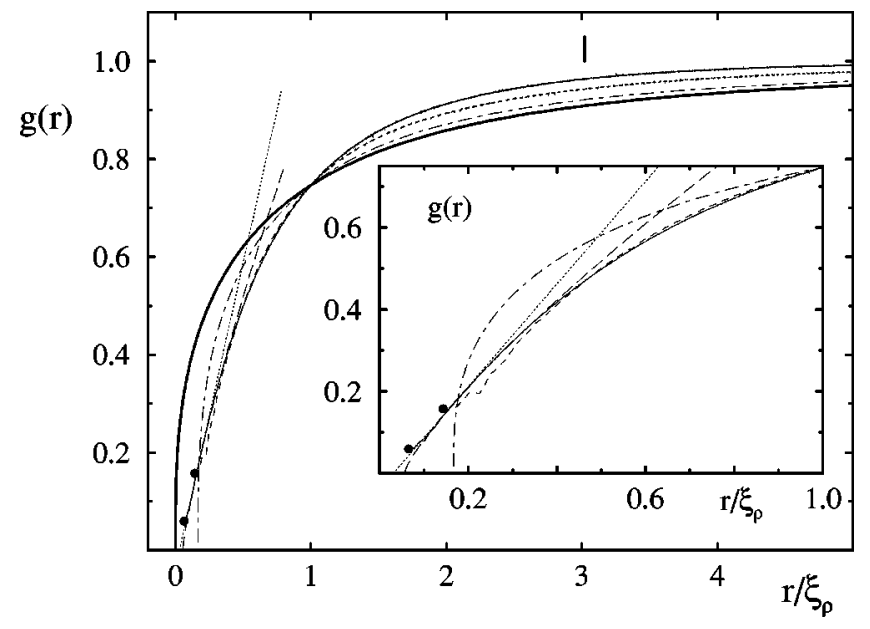

FIG. 3. Pair-correlation functions $g(r)$ versus rescaled distance $r / \xi_{\rho}$ of the BFM for the two densities, $c / c_{*}=25.6$ (thin solid line, $\xi_{\rho}=31.1$ ) and $c / c_{*}=97.7$ (thin dashed line, $\xi_{\rho}=14.0$ ) from [27]. The choice of $\xi_{\rho}$ collapses the curves at $g=0.747$. Circles mark the contact values $g_{d}$. The asymptotic thread PRISM prediction, Eq. (36) (thick solid line), and shifted according to a finite segment size (thin dot-dashed curve) are shown. A small $r$ asymptote, $g$ $=0.99\left[(r-0.855 R) / \xi_{\rho}\right]^{0.80}$ according to Eq. (47) is indicated by a long dashed curve, where $R=2$ in units of the lattice constant is the excluded volume segment diameter of the BFM, and a linear asymptote is given by a dotted line. The vertical bar denotes $\xi_{c} / \xi_{\rho}$ for the lower density. The inset enlarges the small- $r$ region showing the shifted thread PRISM, the power law, and the linear curves with the same line types as in the main part.

asymptotic thread PRISM prediction, Eq. (36), is shifted by a factor indicating that nonasymptotic corrections to $\bar{c}$ cannot be neglected.

A clearer picture of the polymer mesh structure is provided by the pair-correlation function $g(r)$ that is the Fourier transform of $h_{q}$ and asymptotically should follow Eq. (37) in the thread limit, $\sigma, R \ll \xi_{\rho}$ and $\xi_{\rho} \ll \xi_{c} \propto R_{g}$. Figure 3 shows the two $g(r)$ for the above parameters, where $\xi_{\rho}$ is defined by collapsing the simulation data onto the master curve at $g\left(r=\xi_{\rho}\right)=0.747$. Note that this is an unfamiliar definition of $\xi_{\rho}$, which gives values (theoretically) proportional to the standard ones. These values of $\xi_{\rho}$ also produce the collapse of the $h_{q}$ onto a common curve shown in the inset of Fig. 3 and lead to a reasonable collapse of the pair-correlation functions onto a common master curve. Finite-size corrections enter from short distances because of the finite excluded volume segment sizes $R / \xi_{\rho}$. These corrections can also be understood as finite packing fraction corrections. Largedistance deviations from a common curve appear because of the finite chain sizes $\xi_{c} / \xi_{\rho}$. Nevertheless, the short-distance behavior of $g(r)$ provides a sensitive test of the various predictions. The prediction $h_{q} \rightarrow 1 / q^{d}$ would correspond to a logarithmic variation of $g(r \rightarrow 0)$, which appears to be ruled out by the data. Also the thread PRISM prediction, $g(r$ $\rightarrow 0) \propto r^{1 / 3}$, appears incompatible with the data, even if finite segment-size corrections are approximated by a shift of the $r$ origin. The RPA prediction for Gaussian polymers, $g(r$ $\rightarrow 0)-g_{d} \propto r$, of a linear increase in $r$, can describe the data over small intervals (like $0.1<g<0.25$ ) but fails to account for the slight curvature of especially the lower density curve. Moreover, the contact value of the RPA cannot be expected 
to vanish asymptotically if parameters appropriate for a fit to $h_{q}$ are used. An increase in the range of a power-law fit to $g(r)$ at the lower density up to an interval $0.1<g<0.42$ is possible if the following assumption about the paircorrelation function for semidilute solutions is made:

$$
\begin{gathered}
g(r) \rightarrow \bar{g}\left(r / \xi_{\rho}\right) \quad \text { for } \xi_{\rho} \rightarrow \infty, \quad \xi_{\rho} / \xi_{c} \rightarrow 0, \\
\bar{g}(x \ll 1) \propto x^{2 / \nu-d+\omega_{12}(P)},
\end{gathered}
$$

where for dilute cases the same power law with the replacement $\xi_{\rho} \rightarrow \xi_{c}$ can be expected from scaling considerations. This power law would match the scaling law for $g(r)$ for $r$ $\rightarrow 0$ smoothly to the calculated vanishing contact value $g_{d}$ from Eq. (45). Note that such a matching is predicted by PRISM. In Eq. (47), however, the exponent is corrected because the correction to scaling exponent $\omega_{12}(P)$ is taken into account. According to scaling arguments [5,28], there is a term of the form of Eq. (47) present in the intramolecular correlations also, although it is masked by chain-end effects there.

The expected power law for good solutions, $g \propto r^{0.80}$ for $r \ll \xi_{\rho}$ with $\nu=0.588$ and $\omega=0.40$ [1,2,5], is compatible with the simulation data, if a finite shift owing to a finite segment size is anticipated. The power law $h_{q} \rightarrow 1 / q^{2 / \nu+\omega_{12}(P)}$ also is compatible with the data as shown in Fig. 2, but could less be argued on data for $h_{q}$ only.

\section{CONCLUSIONS}

The thread PRISM results derived and discussed here justify earlier phenomenological extensions of RPA-like ex- pressions. The density dependence of the excluded volume parameter is derived from a microscopic incorporation of intermolecular excluded volume and intramolecular connectivity. Various comparisons with rigorous field-theoretic calculations show that leading asymptotic predictions, even for non-mean-field-like situations, are captured correctly in the PRISM integral approach. The correction to scaling exponent, which appears in the molecular mass dependence of the contact value of two polymers, provides a typical example where thread PRISM provides a much better description than mean-field theory but fails to describe all nontrivial correlations. PRISM theory suggests useful concepts like the paircorrelation function $g(r)$ and predicts scalings laws, which provide a framework for the interpretation of data, if the exponents are corrected. Thread PRISM thus turns out rather useful for semidilute solutions, where it explicitly describes the intermolecular correlations of the polymer mesh and results from more rigorous approaches are scarce. Moreover, as PRISM theory is successful for polymer melts, it provides the unique possibility to approach polymer systems at all densities.

\section{ACKNOWLEDGMENTS}

We would like to thank Professor K. S. Schweizer, Professor G. Jannink, Professor K. Binder, Professor A. Milchev, Professor L. Schäfer, and Dr. J. Baschnagel for many valuable discussions, and Dr. E. David for providing the programs to solve the PRISM equations numerically. This work was supported by the Deutsche Forschungsgemeinschaft under Grant Nos. Fu 309/2-1 and Bi 314/17.
[1] P. G. de Gennes, Scaling Concepts in Polymer Physics (Cornell University Press, Ithaca, 1979).

[2] J. D. Cloizeaux and G. Jannink, Polymers in Solution (Oxford Science Publications, Oxford, 1990).

[3] R. Ullman, H. Benoit, and J. S. King, Macromolecules 19, 183 (1986)

[4] G. Jannink, P. Pfeuty, A. Lapp, and J. P. Cotton, Europhys. Lett. 27, 47 (1994).

[5] S. Müller and L. Schäfer, Eur. Phys. J. B 2, 351 (1998).

[6] V. Yamakov, A. Milchev, and K. Binder, J. Phys. II 7, 1123 (1997).

[7] H. Benoit and M. Benmouna, Polymer 25, 1059 (1984).

[8] P. J. Flory and A. M. Bueche, J. Polym. Sci. 27, 219 (1958).

[9] K. S. Schweizer and J. G. Curro, Phys. Rev. Lett. 58, 246 (1987).

[10] K. S. Schweizer and J. G. Curro, Adv. Chem. Phys. 98, 1 (1997)

[11] D. Chandler and H. C. Andersen, J. Chem. Phys. 57, 1930 (1972).

[12] D. Chandler, in Studies in Statistical Mechanics, edited by E. W. Montroll and J. L. Lebowitz (North-Holland, Amsterdam, 1982), Vol. VIII, p. 274.

[13] K. S. Schweizer and J. G. Curro, Macromolecules 21, 3070 (1988); 21, 3082 (1988).
[14] K. S. Schweizer and J. G. Curro, Chem. Phys. 149, 105 (1990).

[15] M. Fuchs and K. S. Schweizer, J. Chem. Phys. 106, 347 (1997).

[16] M. Fuchs, Z. Phys. B 103, 521 (1997).

[17] A. P. Chatterjee and K. S. Schweizer, Macromolecules 31, 2353 (1998).

[18] C. F. Schmidt, M. Bärmann, G. Isenberg, and E. Sackmann, Macromolecules 22, 3638 (1989).

[19] J. P. Hansen and I. R. McDonald, Theory of Simple Liquids (Academic Press, London, 1986).

[20] R. J. Baxter, Aust. J. Phys. 21, 563 (1968).

[21] M. Daoud, J. P. Cotton, B. Farnoux, G. Jannink, G. Sarma, H. Benoit, R. Duplessix, C. Picot, and P. G. de Gennes, Macromolecules 8, 804 (1975).

[22] L. Onsager, Ann. (N.Y.) Acad. Sci. 51, 627 (1949).

[23] T. Shimada, M. Doi, and K. Okano, J. Chem. Phys. 88, 2815 (1988).

[24] G. T. Pickett and K. S. Schweizer, J. Chem. Phys. 110, 6597 (1999).

[25] I. Carmesin and K. Kremer, Macromolecules 21, 2819 (1988).

[26] H. P. Deutsch and K. Binder, J. Chem. Phys. 94, 2294 (1991).

[27] M. Müller and K. Binder (unpublished).

[28] A. Johner, H. Benoit, and J.-F. Joanny, Macromol. Theory Simul. 4, 45 (1995). 